\title{
Lack of association between the HLA region RS7775055 polymorphism and JIA in patients from russia
}

\author{
Victor Malievsky ${ }^{1 *}$, Tatyana Victorova ${ }^{2}$, Ksenia Danilko ${ }^{3}$, Liliia S. Nazarova ${ }^{4}$ \\ From 21st European Pediatric Rheumatology (PReS) Congress \\ Belgrade, Serbia. 17-21 September 2014
}

\section{Introduction}

Juvenile idiopathic arthritis (JIA) refers to a group of conditions involving joint inflammation that first appears before the age of 16 . Juvenile idiopathic arthritis is thought to arise from a combination of genetic and environmental factors. Numerous associations between HLA polymorphisms and JIA subtypes have been reported in multiple populations. The HLA region SNP rs7775055 is a part of the HLA-DRB1*0801-HLA$D Q A 1 * 0401-H L A-D Q B 1 * 0402$ haplotype, which has been shown to increase risk of JIA in UK and non-Hispanic Caucasians US patients. The recent study of Hinks and co-authors also showed the strong evidence of association between rs7775055 and JIA (odds ratio $\left.(\mathrm{OR})=6.01 ; p=3.14 \times 10^{-174}\right)$ in patients from across the US, UK and Germany.

\section{Objectives}

The goal of the study was to test the hypothesis that the HLA region SNP rs7775055 could underlie susceptibility to JIA or its subtypes in patients from Russia.

\section{Methods}

The HLA region SNP rs7775055 was studied in 204 children with JIA and 207 healthy individuals, citizens of the Bashkortostan, Russia using real-time PCR. Statistical analysis was performed using Statistica v.6.0 and SNPStats programs.

\section{Results}

The genotypes distribution was in Hardy-Weinberg equilibrium in both groups. The HLA region SNP rs7775055 did not show an association either with JIA or any of the JIA subtypes in our cohorts. We revealed a trend tendency towards an increase of CC genotype under a recessive model in persistent oligoarthritis cohort, but it was not statistically significant $(p=0,068$, $\mathrm{OR}=6.88$ 95\%CI 0,71-67,02).

\section{Conclusion}

In summary, in this study we found no evidence of association between the HLA region SNP rs7775055 and JIA or its subtypes in patients from Russia.

\section{Disclosure of interest}

None declared.

\begin{abstract}
Authors' details
'Department of Hospital Pediatrics, Bashkir State Medical University, Ufa, Russian Federation. ${ }^{2}$ Biology Department, Laboratory of Human Physiological Genetics, Bashkir State Medical University, Institute of Biochemistry and Genetics, Ufa, Russian Federation. ${ }^{3}$ Biology Department, Central Scientific Research Laboratory, Ufa, Russian Federation. ${ }^{4}$ Biology Department, Bashkir State Medical University, Ufa, Russian Federation.
\end{abstract}

Published: 17 September 2014

doi:10.1186/1546-0096-12-S1-P197

Cite this article as: Malievsky et al.: Lack of association between the HLA region RS7775055 polymorphism and JIA in patients from russia. Pediatric Rheumatology 2014 12(Suppl 1):P197. 